\title{
The Effect of Thyroid Hormone Replacement on Treatment Schedule
}

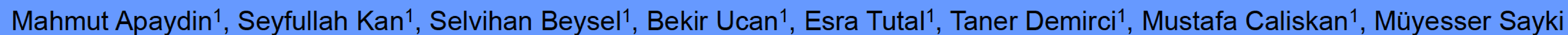 Arslan ${ }^{1}$, Mystafa Ozbek ${ }^{1}$ Erman Cakal ${ }^{1}$ Tuncay Delibasi ${ }^{1,2}$ \\ 1 Diskapi Yildirim Beyazit Teaching and Research Hospital, Department of Endocrinology and Metabolism, Ankara, Turkey \\ ${ }^{2}$ Hacettepe University, School of Medicine (Kastamonu), Department of Internal Medicine, Ankara, Turkey}

\section{Objectives:}

Levothyroxine sodium (LT4) is widely used thyroid hormone to treat hypothyroidism. It is generally recommended to take it in the morning and hungry. Several studies about timing of thyroid hormone administration have shown different effects on TSH and fT4. The aim of this study is to determine the effects of timing of LT4 administration on TSH and the clinical outcomes.

\section{Methods:}

\section{Results:}

\section{Conclusions:}

The study group includes 65 patients with primary hypothyroidism who take a minimum dose of $50 \mathrm{mg}$ levothyroxine sodium 30 minutes before the breakfast. Whether their serum TSH is within the laboratory reference range $(0.5-5,75 \mathrm{mlU} /$ liter $)$ has been examined. We have suggested the patients to take LT4 at bed time instead in the morning and the patients have been observed for three months. Five patients have been excluded from the study for several reasons. At the end of the study parameters such as TSH, fT4, lipid levels, BMI and clinical outcomes have been evaluated.

Compared to the morning intake, LT4 was taken at bedtime were an increase in free T4 level of 0,06+ $0,20(p=0,025)$ and an increase in body weight of $0,76+1,53(p<0,001)$. The TSH levels have shown no significant difference between morning and bedtime intake of LT4 $(p>0,05)$. When the clinical outcomes are evaluated, night sweating in 10 patients $(16,7 \% ; p<0,001)$ and in 8 patients $(13,3 \%$; $p<0,001)$ ) increase in appetite have been observed.

Our study showed that use of LT4 in the morning and at night had similar effects on serum TSH. However, bedtime intake of LT4 has resulted in increase of sweating, appetite and weight. 